\title{
WHO Statement on Caesarean Section Rates
}

\author{
Every effort should be made to provide caesarean \\ sections to women in need, rather than striving \\ to achieve a specific rate
}

\section{Executive summary}

Since 1985, the international healthcare community has considered the ideal rate for caesarean sections to be between $10 \%$ and $15 \%$. Since then, caesarean sections have become increasingly common in both developed and developing countries. When medically justified, a caesarean section can effectively prevent maternal and perinatal mortality and morbidity. However, there is no evidence showing the benefits of caesarean delivery for women or infants who do not require the procedure. As with any surgery, caesarean sections are associated with short and long term risk which can extend many years beyond the current delivery and affect the health of the woman, her child, and future pregnancies. These risks are higher in women with limited access to comprehensive obstetric care.

In recent years, governments and clinicians have expressed concern about the rise in the numbers of caesarean section births and the potential negative consequences for maternal and infant health. In addition, the international community has increasingly referenced the need to revisit the 1985 recommended rate.

\section{Caesarean section rates at the population level}

WHO conducted two studies: a systematic review of available studies that had sought to find the ideal caesarean rate within a given country or population, and a worldwide country-level analysis using the latest available data. Based on this available data, and using internationally accepted methods to assess the evidence with the most appropriate analytical techniques, WHO concludes:

1. Caesarean sections are effective in saving maternal and infant lives, but only when they are required for medically indicated reasons.

2. At population level, caesarean section rates higher than $10 \%$ are not associated with reductions in maternal and newborn mortality rates.

3. Caesarean sections can cause significant and sometimes permanent complications, disability or death particularly in settings that lack the facilities and/or capacity to properly conduct safe surgery and treat surgical complications. Caesarean sections should ideally only be undertaken when medically necessary.

4. Every effort should be made to provide caesarean sections to women in need, rather than striving to achieve a specific rate.

5. The effects of caesarean section rates on other outcomes, such as maternal and perinatal morbidity, paediatric outcomes, and psychological or social well-being are still unclear. More research is needed to understand the health effects of caesarean section on immediate and future outcomes.

\section{Caesarean section rates at the hospital level and the need for a universal classification system}

There is currently no internationally accepted classification system for caesarean section that would allow meaningful and relevant comparisons of CS rates across different facilities, cities or regions. Among the existing systems used to classify caesarean sections, the 10-group classification (also known as the 'Robson classification') has in recent years become widely used in many countries. In 2014, WHO conducted a systematic review of the experience of users with the Robson classification to assess the pros and cons of its adoption, implementation and interpretation, and to identify barriers, facilitators and potential adaptations or modifications.

WHO proposes the Robson classification system as a global standard for assessing, monitoring and comparing caesarean section rates within healthcare facilities over time, and between facilities. In order to assist healthcare facilities in adopting the Robson classification, WHO will develop guidelines for its use, implementation and interpretation, including standardization of terms and definitions. 
For nearly 30 years, the international healthcare community has considered the ideal rate for caesarean sections to be between $10 \%$ and $15 \%$. This was based on the following statement by a panel of reproductive health experts at a meeting organized by the World Health Organization (WHO) in 1985 in Fortaleza, Brazil: "[T]here is no justification for any region to have a rate higher than 10-15\%"(1). The panel's conclusion was drawn from a review of the limited data available at the time, mainly from northern European countries that demonstrated good maternal and perinatal outcomes with that rate of caesarean sections.

Since then caesarean sections have become increasingly common in both developed and developing countries for a variety of reasons (2, 3). When medically justified, caesarean section can effectively prevent maternal and perinatal mortality and morbidity (4). However, there is no evidence showing the benefits of caesarean delivery for women or infants who do not require the procedure. As with any surgery, caesarean sections are associated with short and long term risk which can extend many years beyond the current delivery and affect the health of the woman, her child, and future pregnancies. These risks are higher in women with limited access to comprehensive obstetric care $(5,6,7)$.

The proportion of caesarean sections at the population level is a measure of the level of access to and use of this intervention. It can serve as a guideline for policy-makers and governments in assessing progress in maternal and infant health and in monitoring emergency obstetric care and resource use (8). Over the last few years, governments and clinicians have expressed concern about the rise in the numbers of caesarean section births and the potential negative consequences for maternal and infant health $(9,10,11,12)$. Cost is also a major factor in improving equitable access to maternal and newborn care, as caesarean sections represent a significant expense for overloaded - and often weakened - health systems $(12,13,14)$.

Over the past three decades, as more evidence on the benefits and risks of caesarean section has accumulated, along with significant improvements in clinical obstetric care and in the methodologies to assess evidence and issue recommendations, health care professionals, scientists, epidemiologists and policy-makers have increasingly expressed the need to revisit the 1985 recommended rate $(9,15)$. However, determining the adequate caesarean section rate at the population level - i.e. the minimum rate for medically indicated caesarean section, while avoiding medically unnecessary operations - is a challenging task. To answer this question, WHO conducted two studies: a systematic review of available country-level studies that had sought to find this rate, and a worldwide countrylevel analysis using the latest available data. The process and the results are described in the first part of this Statement.

At the heart of the challenge in defining the optimal caesarean section rate at any level is the lack of a reliable and internationally accepted classification system to produce standardized data, enabling comparisons across populations and providing a tool to investigate drivers of the upward trend in caesarean section.

Among the existing systems used to classify caesarean sections, the 10-group classification (also known as the 'Robson classification') has become widely used in many countries in recent years $(16,17)$. Proposed by Dr Michael Robson in 2001 (18), the system stratifies women according to their obstetric characteristics, thereby allowing a comparison of caesarean section rates with fewer confounding factors. WHO conducted two systematic reviews to assess the value, benefits and potential drawbacks of using this classification to better understand caesarean section rates and trends worldwide. The research process and conclusions are described in detail in the second part of this Statement. 


\section{Caesarean section rates at the population level}

Ecologic studies involve comparisons and analysis of entire populations, rather than individuals. Populations are often defined within geopolitical boundaries, and it is therefore important to differentiate population-based studies from studies of patients in specific health care facilities ('hospitalbased studies').

Healthcare facility rates of caesarean births vary widely depending on differences in the case mix of the obstetric populations they serve, in their capacity and provisions, and in clinical management protocols. Therefore, a population-based recommended caesarean section rate cannot be applied as the ideal rate at the hospital level because of these very differences.

In 2014, WHO conducted a systematic review of the ecologic studies available in the scientific literature, with the objective of identifying, critically appraising, and synthesizing the findings of these studies, which analyse the association between caesarean section rates and maternal, perinatal and infant outcomes (19). At the same time, WHO undertook a worldwide ecologic study to assess the association between caesarean section and maternal and neonatal mortality, using the most recent data available (20). These results were discussed by a panel of international experts at a consultation convened by WHO in Geneva, Switzerland, on 8-9 October 2014. The panel made the following observations:
1. Based on the WHO systematic review, increases in caesarean section rates up to $10-15 \%$ at the population level are associated with decreases in maternal, neonatal and infant mortality (19). Above this level, increasing the rate of caesarean section is no longer associated with reduced mortality. However, the association between higher rates of caesarean section and lower mortality weakened or even disappeared in studies that controlled for socioeconomic factors $(3,21)$. Since it is likely that socioeconomic factors can explain most of the association between increased caesarean section rates and lower mortality in this review, WHO conducted another study to further analyse this aspect.

2. The WHO worldwide ecologic study found that a substantial part of the crude association between caesarean section rate and mortality appears to be explained by socioeconomic factors (20). However, below a caesarean section rate of $10 \%$, maternal and neonatal mortality decreased when caesarean section rates increased. As caesarean section rates increased above $10 \%$ and up to $30 \%$ no effect on mortality rates was observed. The analysis took a longitudinal approach, using country-level data and adjusting for socioeconomic development. This approach may overcome some of the limitations of the cross-sectional studies found in the systematic review but it should be emphasized that ecologic associations do not imply causality.

3. Current data does not enable us to assess the link between maternal and newborn mortality and rates of caesarean section above $30 \%$.

4. Quality of care, particularly in terms of safety, is an important consideration in the analysis of caesarean section rates and mortality. The risk of infection and complications from surgery are potentially dangerous, particularly in settings that lack the facilities and/or capacity to properly conduct safe surgery. 
5. The association between stillbirth or morbidity outcomes and caesarean section rates could not be determined due to the lack of data at the population level. The available ecologic studies analysed mortality indicators only, probably because these are more readily available than maternal and newborn morbidity indicators at the population level. Likewise, psychological and social aspects related to mode of delivery were not considered in the research. Since mortality is a rare outcome, especially in developed countries, future studies must assess the association of caesarean section rates with shortand long-term maternal and perinatal morbidity outcomes (e.g. obstetric fistula, birth asphyxia). These include psychosocial implications regarding the maternal-infant relationship, women's psychological health, women's ability to successfully initiate breastfeeding and paediatric outcomes.

\section{Conclusions}

Based on the available data, and using internationally accepted methods to assess the evidence with the most appropriate analytical techniques, WHO concludes:

1. Caesarean sections are effective in saving maternal and infant lives, but only when they are required for medically indicated reasons.

2. At population level, caesarean section rates higher than $10 \%$ are not associated with reductions in maternal and newborn mortality rates.

3. Caesarean sections can cause significant and sometimes permanent complications, disability or death particularly in settings that lack the facilities and/or capacity to properly conduct safe surgery and treat surgical complications. Caesarean sections should ideally only be undertaken when medically necessary.

4. Every effort should be made to provide caesarean sections to women in need, rather than striving to achieve a specific rate.

5. The effects of caesarean section rates on other outcomes, such as maternal and perinatal morbidity, paediatric outcomes, and psychological or social well-being are still unclear. More research is needed to understand the health effects of caesarean section on immediate and future outcomes. 
At the facilities, it is essential to monitor the rates of caesarean sections taking into account the specific characteristics of the populations that they serve (obstetrical case mix). Currently, there is no standard classification system for caesarean section that would allow the comparison of caesarean section rates across different facilities, cities, countries or regions in a useful and action-oriented manner. As such, it is not yet possible to exchange information in a meaningful, targeted, and transparent manner to efficiently monitor maternal and perinatal outcomes (22).

In 2011, WHO conducted a systematic review of systems used to classify caesarean section, and concluded that the Robson classification is the most appropriate system to fulfil current international and local needs. WHO recommended building upon this to develop an internationally applicable caesarean section classification system (16).

The system classifies all women into one of 10 categories that are mutually exclusive and, as a set, totally comprehensive (see Box 1). The categories are based on five basic obstetric characteristics that are routinely collected in all maternities:

- parity (nulliparous, multiparous with and without previous caesarean section);

- onset of labour (spontaneous, induced or pre-labour caesarean section);

- gestational age (preterm or term);

- foetal presentation (cephalic, breech or transverse); and

- number of foetuses (single or multiple).

The classification is simple, robust, reproducible, clinically relevant, and prospective - which means that every woman admitted for delivery can be immediately classified into one of the 10 groups based on these few basic characteristics. This allows a comparison and analysis of caesarean section rates within and across these groups.
In 2014, WHO conducted a second systematic review of the experience of users with the Robson classification, to assess the pros and cons of its adoption, implementation and interpretation, and to identify barriers, facilitators and potential adaptations (17). WHO convened a panel of experts in Geneva on 8-9 October 2014 to review the evidence. In order to establish a common starting point for comparing maternal and perinatal data within facilities over time and between facilities, the panel made several recommendations:

1. Regardless of their level of complexity, health care facilities should use the Robson classification system for women admitted to give birth.

2. Users of the classification system, while maintaining the original structure necessary for standardized comparisons, may wish to further subdivide the 10 groups and analyse other desirable variables (e.g. epidemiological data, cost, outcomes or indications) within each of the groups, according to their local needs and interests.

3. Reports on the results of the classification should be made publicly available, where possible.

WHO expects that this classification will help health care facilities to:

- optimize the use of caesarean section by identifying, analysing and focusing interventions on specific groups of particular relevance for each health care facility

- assess the effectiveness of strategies or interventions targeted at optimizing the use of caesarean section

- assess the quality of care, clinical management practices and outcomes by group

- assess the quality of the data collected, while raising staff awareness about the importance of the data and its use. 


\section{Box 1: Robson classification}
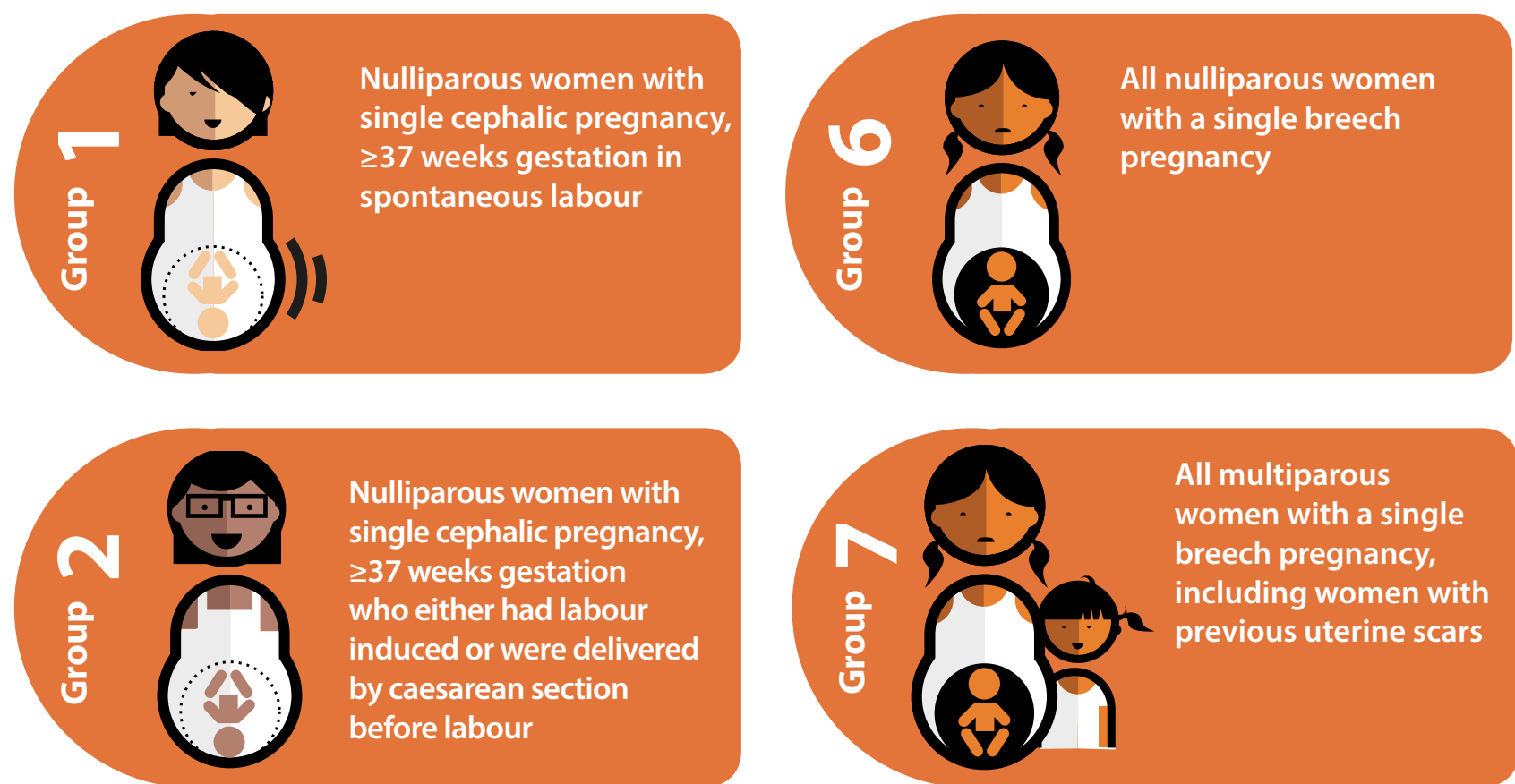

Nulliparous women with single cephalic pregnancy, $\geq 37$ weeks gestation who either had labour induced or were delivered by caesarean section before labour
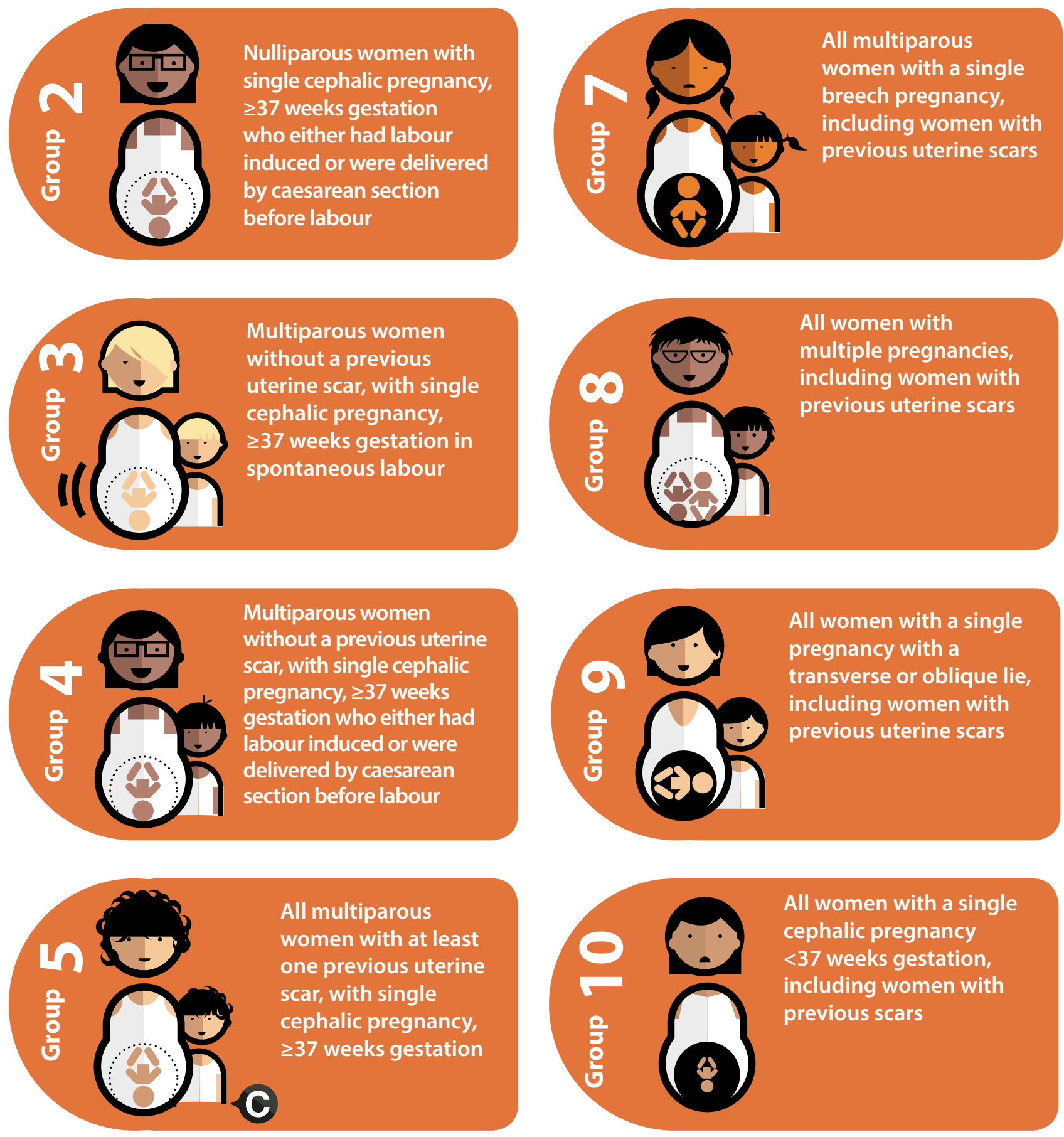
WHO proposes the Robson classification system as a global standard for assessing, monitoring and comparing caesarean section rates within healthcare facilities over time, and between facilities. In order to assist healthcare facilities in adopting the Robson classification, WHO will develop guidelines for its use, implementation and interpretation, including standardization of terms and definitions.

\section{References}

1. Appropriate technology for birth. Lancet. 1985;2(8452):436-7.

2. Vogel JP, Betrán AP, Vindevoghel N, Souza JP, Torloni MR, Zhang J et al. on behalf of the WHO Multi-Country Survey on Maternal and Newborn Health Research Network. Use of the Robson classification to assess caesarean section trends in 21 countries: a secondary analysis of two WHO multicountry surveys. Lancet Global Health 2015;3(5):e260-70.

3. Ye J, Betran AP, Vela MG, Souza JP, Zhang J. Searching for the Optimal Rate of Medically Necessary Cesarean Delivery. Birth. 2014;41(3):237-43.

4. Hannah ME, Hannah WJ, Hewson SA, Hodnett ED, Saigal S, Willan AR. Planned caesarean section versus planned vaginal birth for breech presentation at term: a randomised multicentre trial. Term Breech Trial Collaborative Group. Lancet. 2000;356(9239):1375-83.

5. Lumbiganon $\mathrm{P}$, Laopaiboon M, Gulmezoglu AM, Souza JP, Taneepanichskul S, Ruyan P, et al. Method of delivery and pregnancy outcomes in Asia: the WHO global survey on maternal and perinatal health 200708. Lancet. 2010;375:490-9.

6. Villar J, Carroli G, Zavaleta N, Donner A, Wojdyla $D$, Faundes A, et al. Maternal and neonatal individual risks and benefits associated with caesarean delivery: multicentre prospective study. BMJ. 2007;335(7628):1025.

7. Souza JP, Gulmezoglu A, Lumbiganon P, Laopaiboon M, Carroli G, Fawole B, et al. Caesarean section without medical indications is associated with an increased risk of adverse short-term maternal outcomes: the 2004-2008 WHO Global Survey on Maternal and Perinatal Health. BMC medicine. 2010;8:71.
8. Monitoring emergency obstetric care: a handbook. Geneva, Switzerland: World Health Organization, 2009.

9. Victora CG, Barros FC. Beware: unnecessary caesarean sections may be hazardous. Lancet. 2006;367(9525):1796-7.

10. Steer PJ, Modi N. Elective caesarean sections--risks to the infant. Lancet. 2009;374(9691):675-6.

11. Caesarean section-the first cut isn't the deepest. Lancet. 2010;375(9719):956.

12. Mi J, Liu F. Rate of caesarean section is alarming in China. Lancet. 2014;383(9927):1463-4.

13. Gibbons L, Belizan JM, Lauer JA, Betran AP, Merialdi M, Althabe F. Inequities in the use of cesarean section deliveries in the world. Am J Obstet Gynecol. 2012;206(4):331 e1-19.

14. Connolly ML. High caesarean section figures in Northern Ireland questioned: BBC News Northern Ireland; 2014 [cited 2014]. Available from: http://www.bbc. com/news/uk-northern-ireland-27195161.

15. What is the right number of caesarean sections? Lancet. 1997;349:815-6.

16. Torloni MR, Betran AP, Souza JP, Widmer M, Allen T, Gulmezoglu M, et al. Classifications for cesarean section: a systematic review. PLoS ONE. 2011;6(1):e14566.

17. Betran AP, Vindevoghel N, Souza JP, Gulmezoglu AM, Torloni MR. A Systematic Review of the Robson Classification for Caesarean Section: What Works, Doesn't Work and How to Improve It. PLoS One. 2014;9(6):e97769.

18. Robson MS. Classification of caesarean sections. Fetal and Maternal Medicine Review. 2001;12(1):23-39. 
19. Betran AP, Torloni MR, Zhang J, Ye J, Mikolajczyk R, Deneux-Tharaux $C$ et al. What is the optimal rate of caesarean section at population level? A systematic review of ecologic studies. Reprod Health. 2015;12(1):57.

20. Ye J, Zhang J, Mikolajczyk R, Torloni MR, Gülmezoglu AM, Betrán AP. Association between rates of caesarean section and maternal and neonatal mortality in the 21st century: a worldwide population-based ecological study with longitudinal data. BJOG. 2015 Aug 24 (Epub ahead of print).
21. Althabe F, Sosa C, Belizan JM, Gibbons L, Jacquerioz $F$, Bergel E. Cesarean section rates and maternal and neonatal mortality in low-, medium-, and high-income countries: an ecological study. Birth. 2006;33(4):270-7.

22. Robson M, Hartigan L, Murphy M. Methods of achieving and maintaining an appropriate caesarean section rate. Best Pract Res Clin Obstet Gynaecol. 2013;27:297-308.

\section{human
reproduction
programme
research for impact \\ UNDP.UNFPA.UNICEF·WHO·WORLD BANK}

Further information on WHO's work can be found at: www.who.int/reproductivehealth/

Department of Reproductive Health and Research World Health Organization

Avenue Appia 20, CH-1211 Geneva 27, Switzerland E-mail: reproductivehealth@who.int
WHO/RHR/15.02

(c) World Health Organization 2015

All rights reserved.

Requests for permission to reproduce or translate WHO publications -whether for sale or for non-commercial distribution- should be addressed to WHO Press through the WHO website (www.who.int/about/licensing/copyright_form/ en/index.html).

All reasonable precautions have been taken by the World Health Organization to verify the information contained in this publication. However, the published material is being distributed without warranty of any kind, either expressed or implied. The responsibility for the interpretation and use of the material lies with the reader. In no event shall the World Health Organization be liable for damages arising from its use. 